\title{
ChemComm
}

\section{A generic strategy for pharmacological caging of growth factors for tissue engineering ${ }^{\dagger}$}

\author{
Cite this: Chem. Commun., 2013, \\ 49, 5927 \\ Received 3rd March 2013, \\ Accepted 12th May 2013
}

DOI: $10.1039 / c 3 c c 41616 j$

www.rsc.org/chemcomm

\author{
Maria Karlsson, ${ }^{\text {ab }}$ Philipp S. Lienemann, ${ }^{\text {cd }}$ Natallia Sprossmann, ${ }^{\text {ae }}$ \\ Katharina Heilmann, ${ }^{f}$ Tilman Brummer, ${ }^{\text {efg }}$ Matthias P. Lutolf, ${ }^{d}$ Martin Ehrbar ${ }^{c}$ and \\ Wilfried Weber*abef
}

The caging of small molecules has revolutionized biological research by providing a means to regulate a wide range of processes. Here we report on a generic pharmacological method to cage proteins in a similar fashion. The present approach is of value in both fundamental and applied research, e.g. in tissue engineering.

The trapping of biological compounds in cages and the subsequent controlled uncaging of them at the site of interest allows for a nearly instantaneous manipulation of the bioactive compound concentration. The caging of small signaling molecules, such as second messengers (e.g. calcium and inositol triphosphate), ${ }^{1,2}$ neurotransmitters (e.g. glutamate), ${ }^{3}$ and nucleotides (e.g. ATP and ADP), ${ }^{4,5}$ has revolutionized biological research by providing a means to spatiotemporally regulate and monitor a wide range of cellular processes. Caging based on chemical modification of the molecule using a photo-removable protective group has been successfully applied to a multitude of small signaling molecules. The same strategy has also been applied to chemically synthesizable peptides, ${ }^{6,7}$ however, it is in most cases not compatible with large proteins. Instead, large proteins require a tailored caging procedure for each individual protein of interest, and the caging is often complicated due to difficulties in achieving site-specific chemical modification. Considering the wide range of biological

\footnotetext{
${ }^{a}$ Faculty of Biology, University of Freiburg, Schänzlestrasse 1, 79104 Freiburg, Germany. E-mail: wilfried.weber@biologie.uni-freiburg.de;

${ }^{b}$ Department of Biosystems Science and Engineering, ETH Zurich, Mattenstrasse 26, 4058 Basel, Switzerland

${ }^{c}$ Department of Obstetrics, University Hospital Zurich, Schmelzbergstrasse 12, 8091 Zurich, Switzerland

${ }^{d}$ Institute of Bioengineering, Ecole Polytechnique Fédérale de Lausanne, Station 15, Bld AI 1109, 1015 Lausanne, Switzerland

${ }^{e}$ BIOSS - Centre for Biological Signalling Studies, University of Freiburg, Schänzlestrasse 18, 79104 Freiburg, Germany

${ }^{f}$ ZBSA - Center for Biological Systems Analysis, University of Freiburg, Habsburgerstrasse 49, 79104 Freiburg, Germany

${ }^{g}$ Institute for Molecular Medicine and Cell Research, Stefan-Meier-Strasse 17, 79104 Freiburg, Germany

$\uparrow$ Electronic supplementary information (ESI) available: Details regarding the optimized protein production, MSC mobilization as well as material and methods. See DOI: 10.1039/c3ec41616j
}

signaling processes relying on non-chemically synthesizable proteins, there is a demand for a method enabling the caging of these in a generic manner. Such a method would be an excellent tool for the manipulation of protein-regulated processes, e.g. in developmental biology and tissue engineering. In order to realize this, two separate parts are required: a cage able to trap any protein of choice, and a generically applicable protocol for producing cage-compatible cargo proteins with high yield.

We have developed a generic system enabling pharmacological caging and uncaging of arbitrary proteins equipped with an immunoglobulin (IgG) Fc-tag. The cage was formed by covalently coupling novobiocin to an epoxy-activated agarose matrix via a nucleophilic addition reaction. The anchoring of the cargo protein to the cage was realized using an adaptor protein consisting of the novobiocinbinding domain of the bacterial protein gyrase subunit B (GyrB) fused to the IgG-binding domain $\mathrm{ZZ}$ derived from Staphylococcus aureus protein A (Fig. 1a). The adaptor protein binds to the novobiocin on the cage through its GyrB domain, allowing the ZZ-domain of the adaptor protein to anchor the cargo protein by binding to the Fc-domain. Upon the administration of free novobiocin at pharmacologically relevant concentrations, binding between the adaptor protein and the cage is competitively inhibited, which results in the uncaging of the cargo protein. The rapid uncaging of the protein produces a step function-like release of the bioactive compound, which enables precise temporal regulation of the target process (Fig. 1a).

The cage was characterized in cell culture medium using an Fc-tagged version of the human placental secreted alkaline phosphatase $\left(\mathrm{SEAP}_{\mathrm{Fc}}\right)$ as a human model glycoprotein. Caged $\mathrm{SEAP}_{\mathrm{Fc}}$ was incubated with various concentrations of novobiocin and the amount of uncaged $\mathrm{SEAP}_{\mathrm{Fc}}$ was monitored by measuring the enzymatic activity of the protein over a time period of 5 hours (Fig. 1b). Without novobiocin, only a low level of unspecific uncaging occurs. The addition of novobiocin, either immediately or after two hours, triggered rapid uncaging of the protein. The final activity depended on the novobiocin concentration. A maximum uncaged protein fraction of $70 \%$ was measured at novobiocin concentrations equal to or greater than $60 \mu \mathrm{M}$. The caging efficiency was determined to be $35 \mathrm{ng}$ of $\mathrm{SEAP}_{\mathrm{Fc}}$ per mg of the cage, of which $4.2 \%$ are non-specifically adsorbed. 


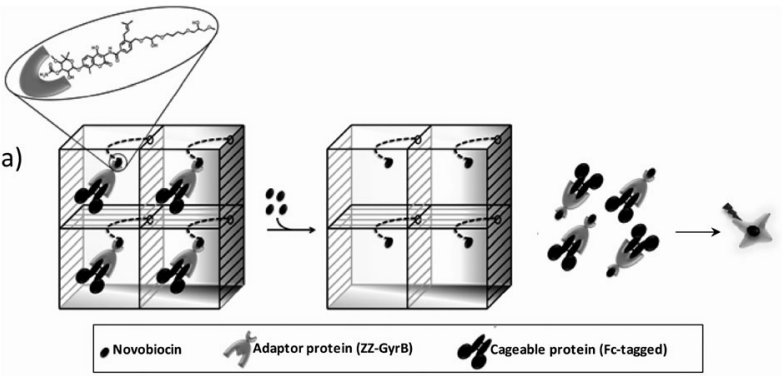

b)

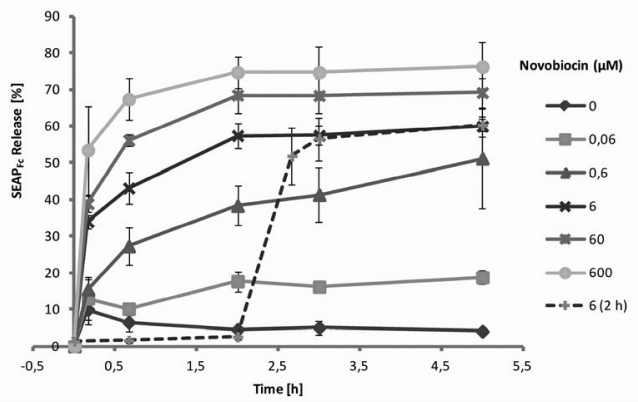

Fig. 1 Design and characterization of the cage. (a) Schematic representation of the cage. The cage is synthesized by covalently functionalizing a mesh-like epoxy-activated agarose resin with novobiocin. An adaptor protein constructed from novobiocin-binding GyrB and the Fc-binding minimal protein A-derived ZZ domain is used to cage an Fccontaining target protein. The administration of free novobiocin competitively inhibits the binding between the adaptor protein and the cage, resulting in uncaging of the cargo protein and regulation of the target process. (b) Dose-dependent uncaging kinetics in cell culture medium. Caged $\mathrm{SEAP}_{\mathrm{FC}}(8 \mathrm{mg}$ cage per $5 \mathrm{ml}$ ) was incubated in DMEM cell culture medium in the presence of various concentrations of novobiocin added immediately or after 2 hours. The activity of uncaged SEAP $\mathrm{Fc}_{\mathrm{F}}$ was determined in the supernatant at the indicated time points. Error bars represent one standard deviation of three experiments.

Growth factor-controlled processes are of fundamental importance in both developmental biology and tissue engineering. One out of the many potential applications of our generic system is the caging of growth factors, which would enable the regulation and thus analysis and engineering of the numerous signaling processes governed by these proteins. To achieve this aim a production protocol applicable to a wide range of growth factors is required in addition to the cage. In order to efficiently engineer an arbitrary growth factor for incorporation into the cage, we devised a generic gene assembly and protein production platform (see ESI $\dagger$ ). The growth factor platform was validated for the manufacturing of cage-compatible variants of the vascular endothelial growth factor $121\left(\mathrm{VEGF}_{\mathrm{Fc}}\right)$, the insulin-like growth factor $1\left(\mathrm{IGF}_{\mathrm{Fc}}\right)$, and the platelet-derived growth factor subunit $\mathrm{B}$ (PDGF-BB $\mathrm{Fc}_{\mathrm{Fc}}$ ), resulting in a protein yield of $950 \mathrm{ng} \mathrm{ml}{ }^{-1}$, $1170 \mathrm{ng} \mathrm{ml}{ }^{-1}$ and $400 \mathrm{ng} \mathrm{m}{ }^{-1}$, respectively. Although the protein expression protocol was not explicitly optimized for each of the growth factors, the production yield was significantly improved for all of them (ESI, † Fig. S1c). This suggests that the optimized production protocol can be generally applied to increase the expression yield of cage-compatible Fc-tagged recombinant proteins.

In order to ensure that the growth factors remain bioactive when equipped with an Fc-tag, bioactivity assays for all produced growth factors were performed. The bioactivity of $\mathrm{VEGF}_{\mathrm{Fc}}$ was confirmed by assessing the $\mathrm{VEGF}_{\mathrm{Fc}}$-induced proliferation of human umbilical vein endothelial cells (HUVECs, Fig. 2a, ref. 8). The bioactivity of IGF1 $1_{\mathrm{Fc}}$ was evaluated by stimulating mouse embryonic fibroblasts (MEFs) with $50 \mathrm{ng} \mathrm{ml}{ }^{-1} \mathrm{IGF}_{\mathrm{Fc}}$ during various time periods resulting in
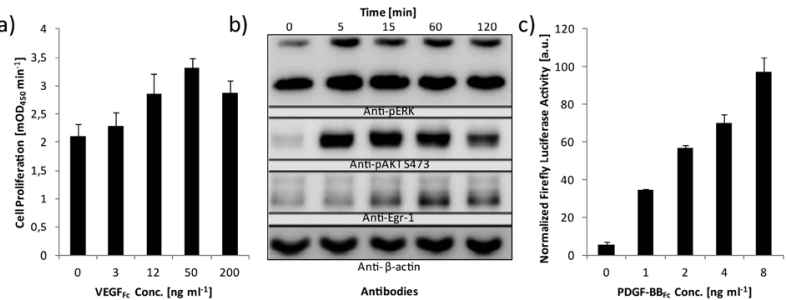

Fig. 2 Validation of growth factor bioactivity. (a) Bioactivity of cageable VEGF $F_{\mathrm{Fc}}$. HUVECS were incubated for $96 \mathrm{~h}$ in the presence of the indicated $\mathrm{VEGF}_{\mathrm{Fc}}$ concentrations prior to quantification of proliferation using the WST-1 assay. Data indicate the mean and the standard deviation of triplicate experiments. (b) Bioactivity of cageable IGF1 $1_{\mathrm{Fc}}$. MEFs were stimulated with $50 \mathrm{ng} \mathrm{ml}^{-1} \mathrm{IGF}_{\mathrm{Fc}}$ for the indicated time periods and the target proteins were detected via western blotting using the indicated antibodies. Anti-pERK; dual phosphorylated extracellular signal regulated kinase, anti-pAKT S473; phospho-Akt (Ser473), anti-Egr-1; early growth response protein 1. (c) Bioactivity of cageable PDGF-BB $\mathrm{FC}_{\mathrm{F}}$. HEK-293T cells were co-transfected with the plasmids for the PDGF signaling pathway coupled to a firefly luciferase output promoter (PDGF-BB receptor, pPDGFR-BB; GAL4-Elk1 fusion protein, pFA2-Elk1; GAL4-responsive firefly luciferase reporter, pFR-Luc). As an internal control, the constitutive renilla luciferase expression vector ( $p G L 4.75)$ was included in the transfection mix. The cells were incubated for $48 \mathrm{~h}$ in the presence of the indicated PDGF-BB $\mathrm{FC}_{\mathrm{F}}$ concentrations prior to quantification of luciferase activity. The PDGF-responsive firefly luciferase activity was normalized to the renilla luciferase activity. Data indicate the mean and the standard deviation of triplicate experiments.

pathway activation as demonstrated by the phosphorylation of ERK and AKT as well as the induction of the immediate early gene product Egr-1 (Fig. 2b, ref. 9 and 10). The bioactivity of PDGF-BB $\mathrm{Fc}_{\mathrm{Fc}}$ was demonstrated using human embryonic kidney cells (HEK-293T) transiently transfected with a PDGF-signaling based luciferase reporter system (Fig. 2c). The observation that all three Fc-engineered growth factors showed bioactivity is in line with previous studies showing that commercially available Fc-tagged proteins represent a highly valuable research tool. ${ }^{11,12}$ In order to analyze whether the adaptor protein changes the binding to the receptor we determined the relative receptor binding affinities of the Fc-engineered growth factors as a function of GyrB-ZZ (ESI, $\nmid$ Fig. S2a-c). As observed from the Scatchard plots, the maximum specific binding and the dissociation constant are comparable for the adaptor-bound and free Fc-growth factors. The recombinant factors without the Fc-tag showed different activity compared to the Fc variants, a factor that is commonly taken into account when working with commercial Fc-tagged growth factors. ${ }^{13}$

Following the successful development of the cage and the establishment of an optimized generic protocol for the production of cage-compatible growth factors, the two were combined to explore the potential to regulate cellular signaling pathways in cell culture by the conditional uncaging of growth factors. For this purpose, caged PDGF-BB $\mathrm{Fc}_{\mathrm{Fc}}$ was added to HEK-293T cells engineered with the PDGF signaling pathway coupled to firefly luciferase. Fig. 3a shows the concentration of uncaged protein and the normalized firefly luciferase activity for cells harvested 7 , 14, or 21 hours post-transfection, cultivated in the absence or presence of novobiocin. The addition of novobiocin resulted in a thirty-fold increased activity for all incubation times investigated, indicating uncaging of bioactive protein and subsequent activation of the PDGF signaling pathway. Cells cultivated in the absence of novobiocin showed a similar activity level to the control cells without a cage, showing that the caged growth 
a)

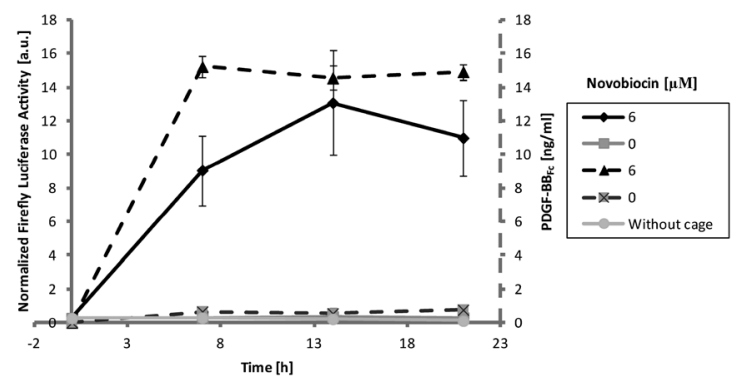

b)

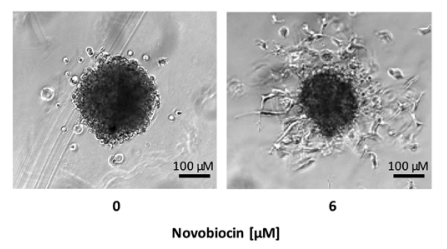

c)

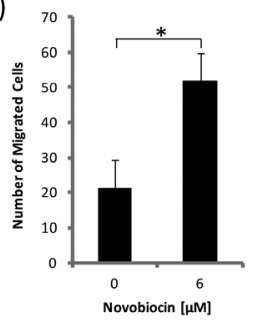

Fig. 3 Inducible uncaging of PDGF-BB $\mathrm{Fc}_{\mathrm{F}}$ for stimulating MSC mobilization. (a) Uncaging of PDGF-BB $B_{F c}$ in cell culture for controlled cell stimulation. Caged PDGF-BB ${ }_{F C}$ (16 mg cage per $\mathrm{ml}$ ) was incubated together with HEK-293T cells transfected with the luciferase-based PDGF reporter system (see Fig. 2c) and cultivated in the presence $(6 \mu \mathrm{M})$ or absence of novobiocin for the indicated time periods prior to quantifying the luciferase activity and the concentration of uncaged PDGF-BB $\mathrm{FC}_{\mathrm{Fc}}$. Data indicate the mean and the standard deviation of triplicate experiments. The solid and dashed lines represent the normalized luciferase activity and the PDGF-BB $\mathrm{FC}_{\mathrm{FC}}$ concentration, respectively. (b) Mobilization of MSCs by inducible growth factor uncaging. Microtissues assembled from MSCs (750 cells) were incorporated into a metalloprotease-sensitive polyethylene glycol-based hydrogel (gel volume: $20 \mu$ l) together with caged PDGF-BB $\mathrm{FC}_{\mathrm{F}}$ (40 mg cage). The hydrogels were incubated in cell culture medium in the presence $(6 \mu \mathrm{M})$ or absence of novobiocin for $16 \mathrm{~h}$. Cell migration was monitored by time-lapse microscopy (see ESIt). Light microscope images of representative spheroids after $16 \mathrm{~h}$ of incubation are shown. (c) Quantification of MSC mobilization. The cells (Fig. 3b) were fixed and stained with DAPI and the total number of migrated cells was determined. The data represent the mean and SEM of three experiments, the asterisk indicates a statistically significant difference to the control with $p<0.05$.

factor did not influence the cellular behavior and that any nonspecific leakage from the cage is negligible (Fig. 3a).

Systems using growth factor concentration gradients to control the migration of mesenchymal progenitor cells (MSCs) are important in the development of cell- and gene-based antitumor therapies. MSCs are known to migrate toward inflamed sites $^{14}$ and have therefore been considered as potential delivery vehicles for the treatment of many diseases, for example cancer. ${ }^{15-17}$ The combination of our cage with MSCs assembled into microtissues enables time-resolved regulation of MSC migration in three dimensions under in vivo-like conditions.

For this purpose, microtissues assembled from MSCs were embedded into a metalloprotease-sensitive polyethylene glycolbased hydrogel ${ }^{18}$ and cultivated together with the cage harboring trapped PDGF- $\mathrm{BB}_{\mathrm{Fc}}$. Controlled uncaging of the protein by the addition of novobiocin allows the uncaged $\mathrm{PDGF} \mathrm{BB}_{\mathrm{Fc}}$ to bind to its cognate extracellular receptor on the MSCs, thereby stimulating the three-dimensional migration of the cells. Fig. 3b shows microscope images of the MSCs and Fig. 3c shows the total number of migrated cells after 16 hours of cultivation together with the cage containing trapped $\mathrm{PDGF}-\mathrm{BB}_{\mathrm{Fc}}$ in the absence or presence of novobiocin.
A movie illustrating the effect of uncaged $\mathrm{PDGF}^{-\mathrm{BB}_{\mathrm{Fc}}}$ on the migration of MSCs can be found in the ESI $\uparrow$ (Video S1).

We have demonstrated a generic approach for the caging and uncaging of arbitrary proteins. The cage along with optimized growth factor production provides a versatile platform for exploring a variety of biological signaling processes through the precisely controlled uncaging of a bioactive compound. The controlled migration of MSCs through regulated uncaging of growth factors illustrates how our system can be used to precisely direct cellular behavior. Considering the tremendous success of caged small signaling molecules in biological research, we believe that the present method will be a valuable asset, not only in developmental biology, tissue engineering and anti-tumor therapy, where the understanding and manipulation of growth factor-controlled signaling processes is of fundamental importance, but also for the regulation of a wide range of additional biological processes of interest in fundamental and applied research.

We thank Aida Kurmanaviciene for technical assistance and Oleg Semenov for help with MSC isolation. This work was supported by the Swiss National Science Foundation (no. CR3213-125426/1 and 310000-116240), the European Union within the framework of the "INTERREG IV Upper Rhine program (project A20), the Initiating and Networking Fund (IVF) of the Helmholtz Association within the Helmholtz Initiative on Synthetic Biology (SO-078) and the excellence initiative of the German Federal and State Governments (EXC-294).

\section{Notes and references}

1 E. A. Finch and G. J. Augustine, Nature, 1998, 396, 753-756.

2 J. W. Walker, A. V. Somlyo, Y. E. Goldman, A. P. Somlyo and D. R. Trentham, Nature, 1987, 327, 249-252.

3 M. Matsuzaki, G. C. Ellis-Davies, T. Nemoto, Y. Miyashita, M. Iino and H. Kasai, Nat. Neurosci., 2001, 4, 1086-1092.

4 J. W. Walker, G. P. Reid, J. A. Mccray and D. R. Trentham, J. Am. Chem. Soc., 1988, 110, 7170-7177.

5 J. H. Kaplan, B. Forbush and J. F. Hoffman, Biochemistry, 1978, 17, 1929-1935.

6 D. S. Miller, S. Chirayil, H. L. Ball and K. J. Luebke, ChemBioChem, 2009, 10, 577-584.

7 A. Nguyen, D. M. Rothman, J. Stehn, B. Imperiali and M. B. Yaffe, Nat. Biotechnol., 2004, 22, 993-1000.

8 M. M. Kampf, E. H. Christen, M. Ehrbar, M. Daoud-El Baba, G. Charpin-El Hamri, M. Fussenegger and W. Weber, Adv. Funct. Mater., 2010, 20, 2534-2538.

9 K. Tsuruzoe, R. Emkey, K. M. Kriauciunas, K. Ueki and C. R. Kahn, Mol. Cell. Biol., 2001, 21, 26-38.

10 T. Arino, K. Tanonaka, Y. Kawahara, T. Maki, N. Takagi, A. Yagi and S. Takeo, Eur. J. Pharmacol., 2008, 580, 298-305.

11 S. M. Brouxhon, S. Kyrkanides, X. Teng, M. Athar, S. Ghazizadeh, M. Simon, M. K. O’Banion and L. Ma, Oncogene, 2013, DOI: 10.1038/ onc.2012.563.

12 S. Gobaa, S. Hoehnel, M. Roccio, A. Negro, S. Kobel and M. P. Lutolf, Nat. Methods, 2011, 8, 949-955.

13 D. M. Czajkowsky, J. Hu, Z. Shao and R. J. Pleass, EMBO Mol. Med., 2012, 4, 1015-1028.

14 E. Spaeth, A. Klopp, J. Dembinski, M. Andreeff and F. Marini, Gene Ther., 2008, 15, 730-738.

15 R. M. Dwyer, J. Ryan, R. J. Havelin, J. C. Morris, B. W. Miller, Z. Liu, R. Flavin, C. O'Flatharta, M. J. Foley, H. H. Barrett, J. M. Murphy, F. P. Barry, T. O'Brien and M. J. Kerin, Stem Cells, 2011, 29, 1149-1157.

16 R. M. Dwyer, S. Khan, F. P. Barry, T. O'Brien and M. J. Kerin, Stem Cell Res. Ther., 2010, 1, 25.

17 M. Studeny, F. C. Marini, R. E. Champlin, C. Zompetta, I. J. Fidler and M. Andreeff, Cancer Res., 2002, 62, 3603-3608.

18 M. Ehrbar, S. C. Rizzi, R. G. Schoenmakers, B. S. Miguel, J. A. Hubbell, F. E. Weber and M. P. Lutolf, Biomacromolecules, 2007, 8, 3000-3007. 